\title{
Ghrelin pretreatment enhanced the protective effect of bone marrow-derived mesenchymal stem cell conditioned medium on lipopolysaccharide-induced endothelial cells injury
}

\section{Shanhui Ge}

Sun Yat-sen University First Affiliated Hospital

Wanmei He

Sun Yat-sen University First Affiliated Hospital

Lishan Zhang

Sun Yat-sen University First Affiliated Hospital

Shan Lin

Sun Yat-sen University First Affiliated Hospital

\section{Yuling Luo}

Sun Yat-sen University First Affiliated Hospital

\section{Qingui Chen}

Sun Yat-sen University First Affiliated Hospital

Mian Zeng ( $\square$ zengmian@mail.sysu.edu.cn )

Sun Yat-sen University First Affiliated Hospital https://orcid.org/0000-0001-9179-902X

\section{Research Article}

Keywords: Ghrelin, mesenchymal stem cells, endothelial cells, AKT/GSK3 $\beta$ signaling pathway

Posted Date: June 22nd, 2021

DOl: https://doi.org/10.21203/rs.3.rs-624712/v1

License: (a) (1) This work is licensed under a Creative Commons Attribution 4.0 International License. Read Full License

Version of Record: A version of this preprint was published at Molecular and Cellular Endocrinology on March 1st, 2022. See the published version at https://doi.org/10.1016/j.mce.2022.111612. 


\section{Abstract \\ Background}

Lung endothelial barrier injury plays a crucial role in the pathophysiology of acute respiratory distress syndrome, and it has been demonstrated that bone marrow-derived mesenchymal stem cells conditioned medium (BMSCs-CM) and ghrelin both exert a potential protective impact. The study aimed to investigate whether ghrelin pretreatment enhanced the protective effect of BMSCs-CM on lipopolysaccharide (LPS)induced endothelial cells injury.

\section{Methods}

BMSCs were isolated from rat bone marrow and used for the experiment after expanded and meeting the phenotypic criteria for defining MSCs identified by flow cytometry. The effect of conditioned medium

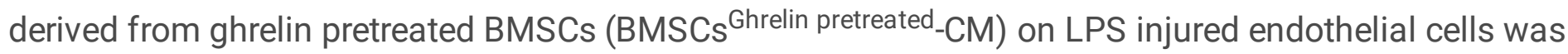
evaluated by examing its impact on the migration capability, apoptosis, and the release of proinflammatory factors (TNF- $\alpha$, IL-1 $\beta$, and IL-6) of endothelial cells. To further clarify the potential mechanisms, the activation of the AKT/GSK3 $\beta$ pathway in endothelial cells was examined by western blot and the gene expression profiles of BMSCs pretreated by ghrelin were examined by RNA sequencing.

\section{Results}

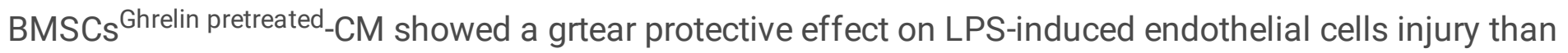
BMSCs-CM by improving cell migration, alleviating apoptosis, and reducing the release of proinflammatory factors of endothelial cells. The mechanism partly related to the activation of the

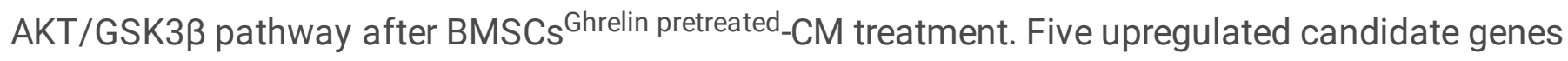
(Wnt5a, S100b, Bmp2, Id4, and Pthlh) were identified in BMSCs after ghrelin treatment which were associated with the activation of AKT pathways and endothelial function.

\section{Conclusions}

Ghrelin pretreatment enhanced the protective effect of BMSCs-CM on LPS-induced endothelial cells injury partly by activating the AKT/GSK3 $\beta$ pathway.

\section{Background}

Acute respiratory distress syndrome (ARDS) is a life-threatening condition which is at least not rare in critically ill patients with high mortality and exerts a substantial disease burden[1]. An international investigation revealed that $10.4 \%$ of ICU patients developed ARDS and the overall mortality of ARDS was 
about 40\%[2]. Therefore, to explore novel therapeutic strategies for ARDS in order to improve prognosis of the condition is of major importance. Lung endothelial barrier injury plays a crucial role in the pathogenesis of ARDS. Under normal condition, a functional alveolar-capillary barrier contributes to the normal gas exchange and removes alveolar edema fluid. Under the pathological condition of ARDS, the damage to capillary endothelial cells and the junctions between cells leads to increased pulmonary vascular permeability and alveolar edema[3]. Thus, lung endothelial barrier injury is a potential therapeutic target for ARDS.

Bone marrow-derived mesenchymal stem cell (BMSC) is a kind of adult stem cells with multidirectional differentiation potential and self-renewal ability[4]. Several studies have reported a beneficial role of BMSCs for treating ARDS[5-7]. A gradually recognized mechanism of the therapeutic effect is that BMSCs provide protective effects on the injured lung endothelial barrier either directly (via secreting soluble factors) or indirectly (via producing extracellular vesicles) [8].Therefore, instead of the cell itself, conditioned medium (CM) or extracellular vesicles derived from BMSCs are considered as a promosing therapeutic option given the cell-free nature [9-11]. Recent studies have focused on how to strengthen the protective effects of BMSCs-derived products. Preconditioning BMSCs with hypoxia[12], cytokine[13], chemical drugs[14], and three-dimensional culture[15] were demonstrated to be capable of changing the secretion profile of BMSCs and play an essential role in the treatments for autoimmune and inflammatory diseases.

Ghrelin was originally discovered as an endogenous hormone involved in the regulation of food intake, bone metabolism, but further researches found its role involved in various physiological functions including cell proliferation and apoptosis[16]. We previously found ghrelin alleviated apoptosis and inflammation in acute lung injury[17]. It has been reported that ghrelin preconditioning both improved the functional survival of MSCs and enhance its curative effect on ischemic heart disease[18]. This suggests ghrelin pretreatment might regulate the the secretion profile of BMSCs and thus improve its therapeutic effect, but it remains unknown whether this also applies to ARDS. Accordingly, our study aimed to investigate whether ghrelin pretreatment enhanced the protective effect of BMSCs-CM on lipopolysaccharide (LPS)-induced endothelial cells injury..

\section{Methods}

\section{Mesenchymal stem cell isolation}

Primary BMSCs were isolated from Rat using the whole bone marrow adherence method. Three-week-old male Sprague-Dawley rats were provided by the laboratory animal center of Sun Yat-sen University and sacrificed after anesthesia. Femur and tibia were separated and their marrow cavities were flushed using sterile phosphate-buffered saline (PBS) to collect bone marrow cells. Flushing fluid was collected in a 15 $\mathrm{mL}$ sterile centrifuge tube and centrifuged at $1000 \mathrm{rpm}$ for 5 minutes. The supernatant was discarded and the cell pellet was resuspended with a complete medium. The cell suspension was removed to the T25 culture flasks and cultured at $37^{\circ} \mathrm{C}$ in $5 \% \mathrm{CO}_{2}$. 


\section{Cell culture}

BMSCs were cultured with low glucose Dulbecco's Modified Eagle Medium (DMEM) and 10\% Foetal Bovine Serum (FBS, Gibco, USA) while the endothelial cells (EA.hy926 cells, purchased from Keygen Biotech Co. (Nanjing, China)) were cultured with high glucose DMEM and 10\% FBS.

\section{Identification for BMSCs}

The third-generation BMSCs were collected and washed with precooled PBA solution (PBS with 1\% BSA (Bovine Serum Albumin)). Then the surface molecules antibodies of CD29 (Cat\#17-0291-80, eBioscience, USA), CD90(Cat\#11-0900-81, eBioscience, USA), and CD45 (Cat\#561867, BD Pharmingen ${ }^{\text {TM }}$, USA) were added and incubated for $30 \mathrm{~min}$ at $4^{\circ} \mathrm{C}$ without illumination. After incubation, BMSCs were washed twice and analyzed by flow cytometry to detect the expression of surface molecules.

\section{Preparation of conditioned medium}

The third-passage BMSCs of the logarithmic growth phase were treated with ghrelin for 24 hours after reaching $80 \%$ confluence, and then the medium was changed into serum-free DMEM. After 24 hours, the conditioned medium was collected, centrifuged at $1000 \mathrm{rpm}$ for 5 minutes to remove cell debris, and stored at $-80^{\circ} \mathrm{C}$ after filtration, labeling as BMSCs ${ }^{\text {Ghrelin pretreated }}$-CM (conditioned medium derived from BMSCs pretreated by ghrelin). Conditioned medium of BMSCs in normal condition was collected in the same way except ghrelin treatment and marking as BMSCs-CM (conditioned medium derived from BMSCs).

\section{Experimental design}

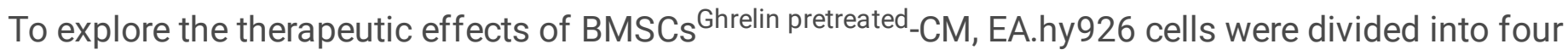
groups, including the control groups (no treatment or treated with DMSO), LPS group (LPS treatment for 24 hours), LPS + BMSCs-CM group (BMSCs-CM was added into the culture system after the endothelial cells were stimulated by LPS for 0.5 hour and co-cultured until 24 hours), and LPS + BMSCs Ghrelin pretreated

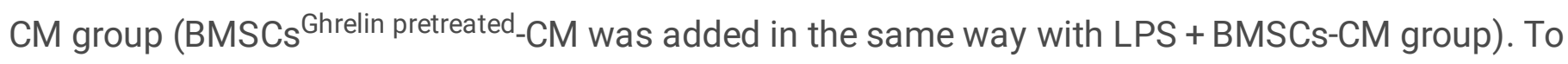
further confirm the role of AKT/GSK3 $\beta$ pathway in the protective effects of BMSCs ${ }^{\text {Ghrelin pretreated_CM on }}$ endothelial cells, LY294002 at a concentration of $10 \mu \mathrm{M}$ was added before LPS treatment. Finally, we

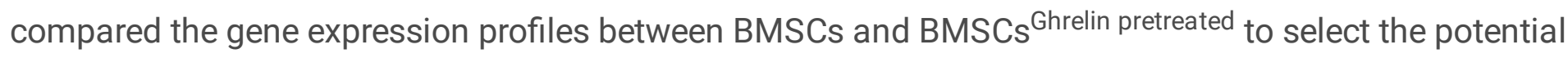
therapeutic molecules.

\section{Cell viability assay}

Cell viability assay was conducted using cell counting kit-8 reagent (CK04, DOJINDO, Japan). Endothelial cells were seeded in 96-well plates (2000 cells per well) and cocultured with conditioned medium derived from BMSCs pretreated with gradient concentrations of ghrelin for 24 hours. BMSCs were seeded in 96well plates (5000 cells per well) and cultured with serum-free DMEM containing gradient concentrations of ghrelin for 24, 48, and 72 hours. For the CCK8 assay, the cells in 96-well plates were added with serum- 
free DMEM containing 10\% CCK8 solution. After incubation at $37^{\circ} \mathrm{C}$ for 2 hours, the absorbance at 450 $\mathrm{nm}$ was measured with a microplate reader (Sunrise, TECAN)

\section{Scratch wound healing assay}

Endothelial cells were seeded in 6-well plates $\left(5 \times 10^{4}\right.$ cells per well) and grew until $90 \%$ confluence. Scratch was made by a $200 \mu \mathrm{L}$ sterile pipette tip and PBS was used to wash off the exfoliated cells. Then serum-free DMEM, LPS, and conditioned medium were added as described before. The photos of scratches were taken at 0 hour and 24 hours, respectively. The areas of scratches were measured and analyzed using Image $\mathrm{J}$ software. Wound healing rates $(\%)=($ scratch area at $0 \mathrm{~h}$ - scratch area at $24 \mathrm{~h}) /$ scratch area at $0 \mathrm{~h} \times 100 \%$.

\section{Mitochondrial membrane potential assay}

Endothelial cells were seeded in 6-well plates $\left(5 \times 10^{4}\right.$ cells per well) and treated as described before. Mitochondrial membrane potential was detected using a Mitochondrial membrane potential assay kit with JC-1 (Beyotime, China). Endothelial cells were washed once using PBS and then incubated with a $\mathrm{JC}-1$ probe $(10 \mu \mathrm{M})$ at $37^{\circ} \mathrm{C}$ for 20 minutes. After incubation, cells were washed twice with JC- 1 staining buffer to remove excess JC-1 probe. The green and red fluorescence intensity of endothelial cells was observed under a fluorescent microscope (Leica DMI8) and analyzed by Image J software.

\section{Apoptosis assay by flow cytometry}

Apoptosis rates of endothelial cells were detected by a FITC Annexin V Apoptosis Detection Kit I (BD Pharmingen $^{\mathrm{TM}}$, USA). Endothelial cells were collected and centrifuged at 1000 rpm for 5 minutes. Then cells were washed twice with precooled PBS and resuspended in Annexin $\mathrm{V}$ binding buffer at a concentration of $1 \times 10^{6}$ cells $/ \mathrm{ml}$. FITC Annexin V and Propidium lodide (PI) staining solution were added, blended gently, and incubated with endothelial cells at RT $\left(25^{\circ} \mathrm{C}\right)$ for 15 minutes without illumination. The percentages of Annexin $\mathrm{V}$ and PI-positive cells were analyzed by flow cytometry within one hour.

\section{Western blotting}

Endothelial cells were washed with PBS and treated with RIPA lysis solution contained PMSF (Beyotime, China) and Phosphatase Inhibitor (CoWin Biosciences, China) at a final concentration of $1 \mathrm{mM}$ to extract protein. The cell lysates were centrifuged at $14000 \mathrm{~g}$ for 5 minutes at $4^{\circ} \mathrm{C}$ and the supernatant was collected. Proteins were quantified with a BCA Protein Assay Kit (CoWin Biosciences, China) and separated using 10\% SDS- PAGE (sodium dodecyl sulfate-polyacrylamide gel electrophoresis). Then proteins were transferred to the Immobilon-P Transfer Membrane (Millipore, USA) and blocked with 2\% BSA solution at RT $\left(25^{\circ} \mathrm{C}\right)$ for 1 hour. After washed by TBST (Tris-buffered saline with $0.1 \%$ Tween 20 ) three times, the membranes were incubated with primary antibodies as follows: anti-AKT (1:1000, Cat\#4691S, Cell Signaling Technology, USA), anti-phospho-AKT (1:1000, Cat\#4060S, Cell Signaling Technology, USA), anti-GSK3 $\beta$ (1:1000, Cat\#5676S, Cell Signaling Technology, USA), anti-phospho-GSK3 $\beta$ (1:1000, Cat\#9322S, Cell Signaling Technology, USA), anti-Bax (1:1000, Cat\#5023S, Cell Signaling Technology, USA), anti-Bcl-2 (1:1000, Cat\#4223S, Cell Signaling Technology, USA), and anti-GAPDH 
(1:1000, Cat\#5174S, Cell Signaling Technology, USA). Secondary antibodies conjugated to horseradish peroxidase (1:5000, Cat\#7074S, Cell Signaling Technology, USA) were added and incubated with the membranes for 1 hour at RT $\left(25^{\circ} \mathrm{C}\right)$. Finally, the protein bands were detected by the ECL chemiluminescence method.

\section{Quantitative real-time PCR}

Total RNA was extracted from endothelial cells using RNA-Quick Purification Kit (RN001, ESscience, China) and the purity was detected by the A260/A280 ratios. Then $1000 \mathrm{ng}$ RNA was reverse transcribed to cDNA using cDNA Synthesis SuperMix (NovoProtein, China). Quantitative real-time PCR was performed by SYBR qPCR Mix (NovoProtein, China) to analyze the expression of GAPDH, interleukin-6 (IL-6), tumor necrosis factor-a (TNF-a), and interleukin-1 $\beta$ (IL-1 $\beta$ ) (primers of each gene were shown in Table 1).

Table 1

primers designed for qPCR

\begin{tabular}{|lll|}
\hline Genes & Sequence & Length (bp) \\
\hline GAPDH & F: 5'-AGGGCATCCTGGGCTACACT-3' & 154 \\
& R: 5'-TTGCTGTAGCCAAATTCGTTGT-3' \\
\hline TNF-a & F: 5'-CACAGTGAAGTGCTGGCAAC-3' & 185 \\
\hline & R: 5'-AGGAAGGCCTAAGGTCCACT-3' \\
\hline IL-6 & F: 5'-TGAGGAGACTTGCCTGGT-3' & 186 \\
\hline & R: 5'-GGGTCAGGGGTGGTTATT-3' & \\
\hline IL-1ß & F: 5'-ATCAGCACCTCTCAAGCAG-3' & 139 \\
\hline & R: 5'-AGTCCACATTCAGCACAGG-3' \\
\hline
\end{tabular}

\section{RNA sequencing analysis}

Total RNA was extracted from the BMSCs and BMSCs pretreated with ghrelin and residual DNA was removed using DNase I. The RNA was successively purified by RNA Clean XP magnetic beads and enriched by Oligo ( $\mathrm{dT}$ ) magnetic beads. The enriched product (mRNA) was divided into small fragments (150-300 nt) and cDNA was synthesized using Reverse Transcriptase SuperScript II, RNaseH, and DNA polymerase, sequentially. The cDNA was purified by AmPure XP Bead and repaired the terminal. Then A was added to the $3^{\prime}$ and the sequencing joints were connected. The cDNA library was established after purification and qPCR amplification. The qualified library was pooling according to the effective concentration and the amount of target data, and the sequencing is conducted on the HiSeq platform according to PE 150 strategy. The TPM (Transcripts Per Million) was utilized to calculate the expression of genes and those with $\|$ log2 (fold change) $\mid \geq 1$ and $p$-value $<0.05$ were considered statistically significant genes. Gene Ontology (GO) (http://www.geneontology.org/) and KEGG 
(http://www.genome.jp/kegg/) analysis were performed to discuss the functions and enriched pathways of statistically significant genes.

\section{Statistical analysis}

Continuous data were expressed as mean \pm standard deviation (). One-way ANOVA and post hoc tests were utilized to compare differences among the groups. All the experimental data were statistically analyzed by SPSS 25.0. A p-value less than 0.05 was considered as statistically significant.

\section{Results}

\section{Surface markers identification of BMSCs}

At passage 3, BMSCs adhered to the plate with uniform morphology and spindle shape (Fig. 1a). According to the flow cytometry, the surface markers CD29 $(99.84 \pm 0.09 \%)$ and CD90 $(98.24 \pm 0.49 \%)$ were highly expressed in BMSCs while CD45 (4.21 $\pm 0.33 \%)$ was lowly expressed (Fig. 1b, c, d).

\section{Optimum concentration selection of ghrelin}

To determine the optimal concentration of ghrelin for pretreatment, we first detected the effect of different concentrations of ghrelin on the viability of BMSCs under a serum deprivation condition. When cultured in a serum-free medium, the viability of BMSCs decreased in a time-dependent manner, and the best viability of BMSCs was observed when the cells were treated by $100 \mathrm{nM}$ and $1000 \mathrm{nM}$ ghrelin (Fig. 2a). We further collected the conditioned medium from BMSCs stimulated by different concentrations of ghrelin for 24 hours and co-cultured with endothelial cells. The results showed that the best viability of endothelial cells was observed when co-cultured with conditioned medium derived from $100 \mathrm{nM}$ ghrelin pretreated BMSCs (Fig. 2b). According to these results, we chose the $100 \mathrm{nM}$ ghrelin as the appropriate concentration for the following experiments.

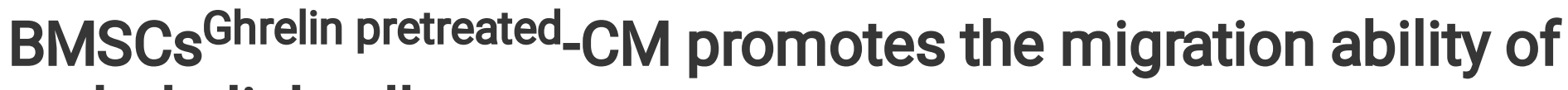 endothelial cells}

Scratch wound healing assay was utilized to analyze the migration ability of endothelial cells. As exhibited in Fig. 3a b, LPS significantly inhibited endothelial cells migration, and BMSCs-CM treatment could enhance endothelial cells migration. Compared with the BMSCs-CM, BMSCs ${ }^{\text {Ghrelin pretreated_CM }}$ better-promoted wound closure after scratching.

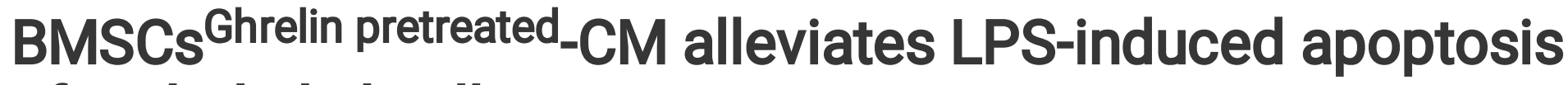 of endothelial cells}




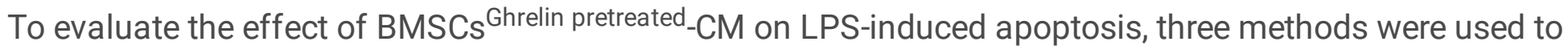
detect the apoptosis of endothelial cells. As shown in Fig. 3c d, LPS treatment significantly decreased the mitochondrial membrane potential of endothelial cells ( $\mathrm{p} \otimes 0.05$ ). .Compared with the LPS group, the mitochondrial membrane potential in BMSCs-CM and BMSCs ${ }^{\text {Ghrelin pretreated_CM groups was lower }(\mathrm{p}<}$ 0.05). However, no significant difference was found between BMSCs-CM and BMSCs ${ }^{\text {Ghrelin pretreated-CM }}$ groups.

Next, AnnexinV and PI dual staining was used to label the apoptotic cells (Fig. $3 e \sim f$ ). In comparison with the control group, LPS induced obvious apoptosis in endothelial cells ( $7.33 \%$ versus $50.93 \%$, respectively, $\mathrm{p}<0.01$ ). The percentages of apoptotic cells in BMSCs-CM and BMSCs ${ }^{\text {Ghrelin pretreated_CM groups were }}$ higher than that of the LPS group. Moreover, the apoptotic rate of endothelial cells in the BMSCs ${ }^{\text {Ghrelin }}$ pretreated_CM group was significantly lower compared with the BMSCs-CM group(38.94\% versus $46.59 \%$, respectively, $\mathrm{p}<0.01)$. Furthermore, the results of western blotting showed that BMSCs ${ }^{\text {Ghrelin pretreated }}$-CM reduced the expression of Bax protein and increased the expression of $\mathrm{Bcl}-2$ protein compared with the LPS group and BMSCs-CM group (Fig. $3 g \sim i$ ).

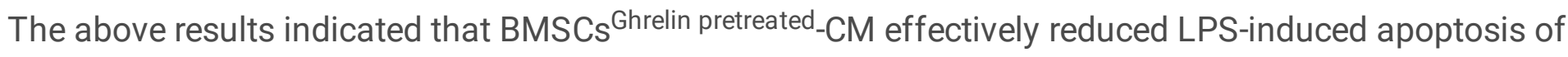
endothelial cells.

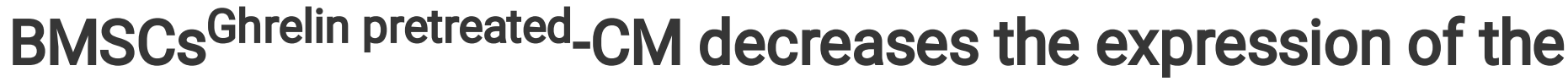 inflammatory cytokines of endothelial cells}

As the results of qPCR showed (Fig. 4), inflammatory cytokines TNF-a, IL-1 $\beta$, and IL-6 exhibited increased expression after LPS treatment, and the expression of above cytokines was downregulated in BMSCs-CM

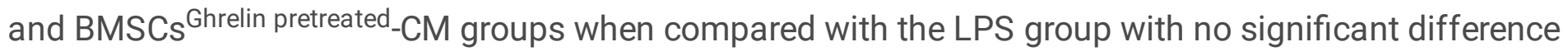

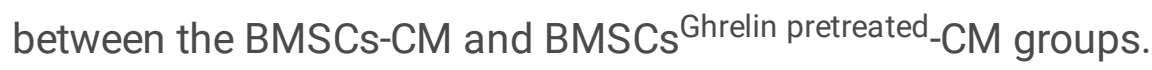

\section{AKT /GSK3 $\beta$ signaling pathway mediated the protective effect of BMSCs ${ }^{\text {Ghrelin pretreated }}$-CM}

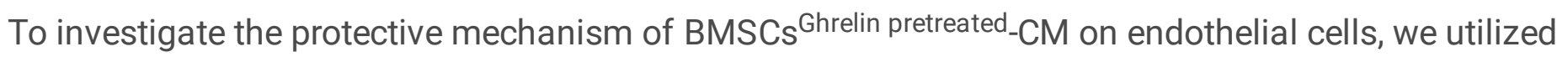
western blotting to assess the phosphorylation of AKT and GSK3 $\beta$ protein. LPS decreased the phosphorylation of AKT and GSK3 $\beta$ protein compared with the control group, whereas BMSCs ${ }^{\text {Ghrelin }}$ pretreated_CM significantly increased the phosphorylation of AKT and GSK3 $\beta$ protein (Fig. 5a,b,c). Then, $\mathrm{PI3K} / \mathrm{AKT}$ inhibitor LY294002 was added to block activation of AKT signaling pathway, we found that

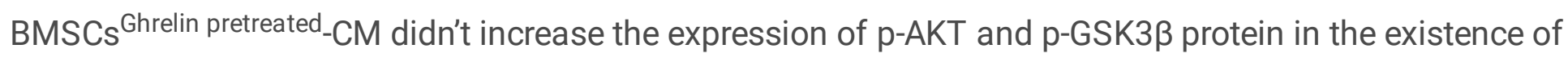

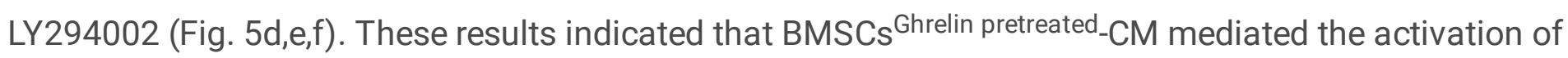
the AKT /GSK3 $\beta$ signaling pathway. 


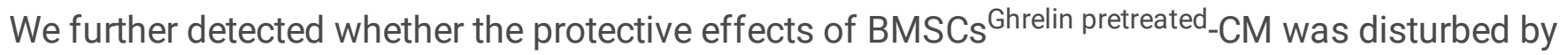
LY294002. We treated endothelial cells with LY294002 1h before LPS stimulation, and the anti-apoptotic

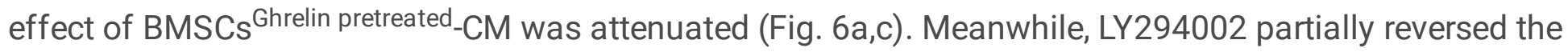

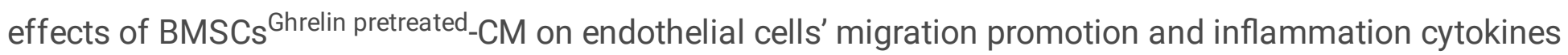

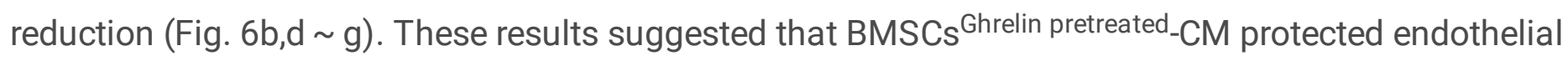
cells from LPS induced injury in part through the activation of the AKT / GSK3 $\beta$ signaling pathway.

\section{Influence of ghrelin on the genes expression profile of BMSCs}

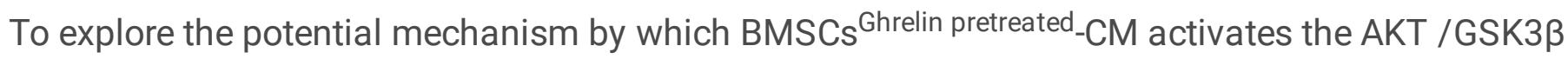
signaling pathway in endothelial cells, we performed transcriptome sequencing on BMSCs with and without ghrelin treatment and analyzed the relationship between differentially expressed genes (DEGs) and the function of endothelial cells. In total, we found 88 upregulated genes and 405 downregulated genes in the ghrelin group (Fig. 7a). The GO and KEGG analysis exhibited that DEGs were mainly related to the biological processes including cytokines and chemokines interaction, osteoclast differentiation, immune responses, and defenses to microbial infections(Fig. $7 b, c)$. We further discovered 5 upregulated DEGs both associated with activation of AKT signaling pathways and endothelial function: Wnt5a, S100b, Bmp2, Id4, and Pthlh (Fig. 7d), most of which encoded secreted proteins.

\section{Discussion}

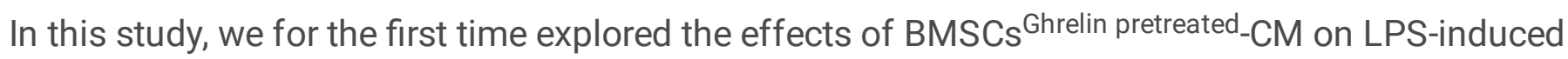
endothelial injury. Our results showed that ghrelin pretreatment signifiantly enhance the protective effects of BMSCs-CM on LPS-induced endothelial barrier dysfunction, which was partly mediated by activation of the AKT/GSK3 $\beta$ signaling pathways. Our study provided the theoretical basis for a new therapeutic strategy of ARDS.

Currently, the mortality of ARDS remains high, about $40 \%$, and the main therapeutic strategies were lungprotective mechanical ventilation and anti-infection treatment, and there is lack of intervention targeting at the pathogenesis[19]. The damage of alveolar epithelial cells and alveolar endothelial cells caused by various pathogenic factors is the key process of the ARDS. LPS, also known as endotoxin, is a constituent of the outer wall of Gram-negative bacteria cells and is often used to induce ARDS injury model[20]. In this study, EA.hy926 cells were treated with LPS to induce ARDS-related endothelial injury, and we found that LPS significantly increased apoptosis, elevated the expression level of TNF- $a$, IL-1 $\beta$, and IL-6, and impaired the migration ability of the endothelial cells. These results indicated that LPS treatment effectively induced endothelial dysfunction.

Known as pluripotent stem cells with immunomodulatory capacity, MSCs secrete cytokines, growth factors, and bioactive factors to reduce the inflammatory response and repair damaged tissues. The 
conditioned medium derived from MSCs was demonstrated to attenuated the endothelial barrier dysfunction in different conditions, including hypoxia[21], ischemia[22], diabetes[23], and wound healing[24]. In this study, we found that BMSCs-CM reduced the apoptosis and inflammatory factors expression, and improved the migration ability of endothelial cells, which was consistent with previous studies.

Ye et al[25] demonstrated that BMSCs highly expressed growth hormone secretagogue receptor (GHSR), which was the receptor of ghrelin. Ghrelin promoted the viability, osteogenesis, and chondroblast differentiation of MSC by regulating the ERK1/2 signaling pathway[25, 26]. As reported, ghrelin preconditioning enhanced the therapeutic effects of MSCs[18], then we assumed that ghrelin preconditioning might also impact the effects of MSCs on the endothelial injury. First, we detected the viability of endothelial cells co-cultured with conditioned medium derived from different concentrations of ghrelin pretreated BMSCs with LPS treatment, and $100 \mathrm{nM}$ ghrelin-pretreated BMSCs-CM better improved the viability of endothelial cells compared with BMSCs-CM. Then, therapeutic effects of BMSCs ${ }^{\text {Ghrelin }}$ pretreated_CM on endothelial cell injury under LPS attack were observed, and BMSCs ${ }^{\text {Ghrelin }}$ pretreated_CM remarkably reduced the apoptosis and improved the migration of endothelial cells in comparison with BMSCs-CM. Taken together, our results suggested that ghrelin preconditioning enhanced the ability of BMSCs-CM to repair the endothelial injury.

AKT/GSK3 $\beta$ signaling pathway plays an essential role in regulating the process of apoptosis, inflammation, proliferation, and migration[27, 28]. Si et al [29]found that adrenomedullin alleviated the apoptosis induced by hypoxia and serum deprivation via activating the AKT/GSK3 $\beta$ signaling pathway. In LPS-stimulated macrophages, the activation of the PI3K/AKT/GSK3 $\beta$ pathway reduced the nuclear translocalization of NF-KB and then downregulated the expression of IL-6 and IL-12[30]. Furthermore, AKT/GSK3 $\beta$ pathway was confirmed to mediate the repair of endothelial barrier dysfunction in ARDS[31]. Therefore, we assessed the activation of the AKT/GSK3 $\beta$ pathway using western blotting. The results

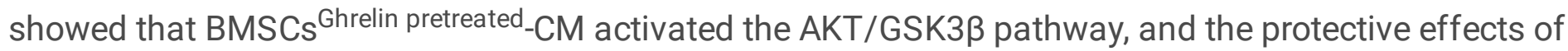

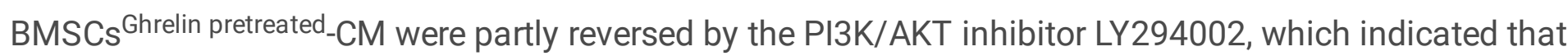

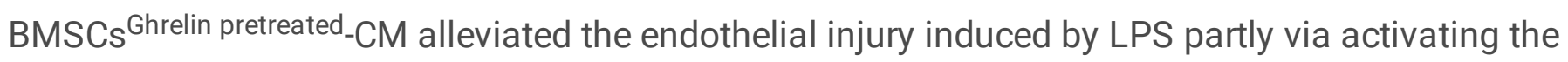
AKT/GSK3 $\beta$ pathway.

Finally, we tried to investigate the change of gene expression profile in BMSCs pretreated by ghrelin using RNA-seq sequencing. According to our previous results, we hypothesized that ghrelin preconditioning promotes the secretion of certain proteins which acts on endothelial cells and activates the AKT/GSK3 $\beta$ pathway to exert protective effects. We discovered five upregulated genes in ghrelin preconditioning BMSCs which were both related to the activation of the AKT pathway[32-37] and the endothelial function: Wnt5a, S100b, Bmp2, Id4, and Pthlh. Wnt5a, known as secretory protein, is an important regulator of the non-classical Wnt pathway and plays a double-sided role on endothelial cells. Zhang et al [38]found that exosomes derived from MSCs reduced hypoxia-induced apoptosis of rat pulmonary artery endothelial cells by increasing the expression of Wnt5a protein while Breton-Romero et al[39] 
demonstrated that the activation of Wnt5a contributed to the endothelial dysfunction. S100b is a secretory calcium-binding protein that was initially found to be secreted by glial cells, and subsequently detected in non-neural tissues such as chondrocytes and adipose tissue[40]. Exogenous S100b protein promoted angiogenesis by facilitating the release of VEGF and tube formation of human umbilical vein endothelial cells[41]. Bmp2 (bone morphogenetic protein 2), a member of the TGF- $\beta$ superfamily, regulated bone formation through autocrine and paracrine mechanisms. Recent studies reported that Bmp2 also had an impact on endothelial cells and angiogenesis. Zuo et al [42]revealed that Bmp2 promoted the proliferation, migration, and angiogenesis of human umbilical vein endothelial cells through p38, ERK, and AKT/mTOR pathways. In addition, ID4 (DNA binding inhibitor 4) and Pthlh (parathyroid hormone-associated protein) were mainly related to the pro-angiogenesis ability of cancer[37, 43]. Taken together, our results indicated that ghrelin preconditioning upregulated the expression of Wnt5a, S100b, Bmp2, Id4, and Pthlh, which probably contribute to the improvement of endothelial dysfunction.

\section{Limitations}

Our study has several limitations. First, a endothelial cell line but not primary endothelial lung cells was utilized to simulate the pulmonary endothelial system in vitro. Moreover, the pathway inhibitor-LY294002 used in this study might has off target effects, and siRNA methodology would be a better method to

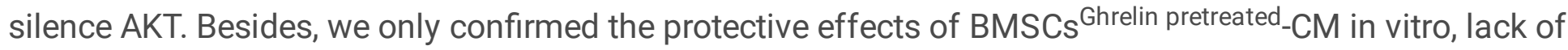
further confirmatory animal experiments.

\section{Conclusion}

In summary, we demonstrated that ghrelin pretreatment strengthen the protective effects of BMSCs-CM by inhibiting LPS-induced apoptosis and inflammation, and improving the migration of endothelial cells in vitro via activation of AKT/GSK3 $\beta$ pathway, which might be associated with ghrelin-induced upregulation of Wnt5a, S100b, Bmp2, Id4, and Pthlh genes in BMSCs. Nevertheless, the detailed mechanism involving in the therapeutic effects of ghrelin-pretreated BMSCs-CM needs further studies to elucidate.

\section{Abbreviations}

ARDS, Acute respiratory distress syndrome; BMSCs, Bone marrow-derived mesenchymal stem cells; MSCs, Mesenchymal stem cells; BMSCs-CM, bone marrow-derived mesenchymal stem cells conditioned

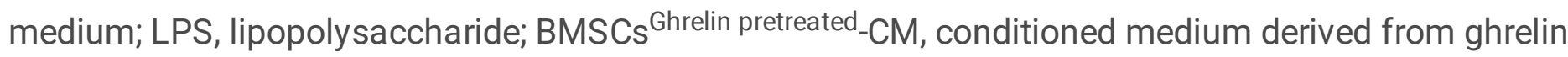
pretreated BMSCs; DMEM, Dulbecco's Modified Eagle Medium; FBS, Foetal Bovine Serum; CCK8, cell counting kit-8; DEGs, Differentially expressed genes; Bmp2, bone morphogenetic protein 2; ID4, DNA binding inhibitor 4; Pthlh, Parathyroid hormone-associated protein. 


\section{Declarations}

\section{Availability of data and material}

The datasets used or analyzed during the current study are available from the corresponding author on reasonable request.

\section{Ethics approval and consent to participate}

The study was approved by the Ethics Committee of the First Affiliated Hospital, Sun Yat-sen University (Grant number: [2019]315).

Consent for publication: Not applicable

\section{Funding}

This work was supported by National Natural Science Foundation of China (Grant number 81670066); Guangdong Basic and Applied Basic Research Foundation (Grant number 2019A1515011198); Major Science and Technology Planning Project of Guangdong Province, China (Grant number 2016A020216009).

\section{Acknowledgements}

Thanks Prof. Lihua Zhou for providing the experimental spaces and instruments.

\section{Disclosure}

The authors declare that there is no conflict of interest.

\section{Author Contributions}

MZ conceptualized and designed the study. SG and WH conducted the experiments, analyzed the study data, and wrote the initial draft. LZ and SL did the data curation. YL performed some of the experiments. $\mathrm{QC}$ revised the manuscript.

\section{References}

1. McNicholas BA, Rooney GM, Laffey JG. Lessons to learn from epidemiologic studies in ARDS. Curr Opin Crit Care. 2018;24(1):41-8.

2. Bellani G, Laffey JG, Pham T, Fan E, Brochard L, Esteban A, Gattinoni L, van Haren F, Larsson A, McAuley DF, et al. Epidemiology, Patterns of Care, and Mortality for Patients With Acute Respiratory Distress Syndrome in Intensive Care Units in 50 Countries. JAMA. 2016;315(8):788-800.

3. Vassiliou AG, Kotanidou A, Dimopoulou I, Orfanos SE. Endothelial Damage in Acute Respiratory Distress Syndrome. Int J Mol Sci 2020, 21 (22). 
4. Ding DC, Shyu WC, Lin SZ. Mesenchymal stem cells. Cell Transplant. 2011;20(1):5-14.

5. Tang XD, Shi L, Monsel A, Li XY, Zhu HL, Zhu YG, Qu JM. Mesenchymal Stem Cell Microvesicles Attenuate Acute Lung Injury in Mice Partly Mediated by Ang-1 mRNA. Stem Cells. 2017;35(7):184959.

6. Hao Q, Gudapati V, Monsel A, Park JH, Hu S, Kato H, Lee JH, Zhou L, He H, Lee JW. Mesenchymal Stem Cell-Derived Extracellular Vesicles Decrease Lung Injury in Mice. J Immunol. 2019;203(7):1961-72.

7. Li L, Dong L, Zhang J, Gao F, Hui J, Yan J. Mesenchymal stem cells with downregulated Hippo signaling attenuate lung injury in mice with lipopolysaccharide-induced acute respiratory distress syndrome. Int J Mol Med. 2019;43(3):1241-52.

8. Liang X, Ding Y, Zhang Y, Tse HF, Lian Q. Paracrine mechanisms of mesenchymal stem cell-based therapy: current status and perspectives. Cell Transplant. 2014;23(9):1045-59.

9. Vizoso FJ, Eiro N, Cid S, Schneider J, Perez-Fernandez R. Mesenchymal Stem Cell Secretome: Toward Cell-Free Therapeutic Strategies in Regenerative Medicine. Int J Mol Sci 2017, 18(9).

10. Wang H, Zheng R, Chen Q, Shao J, Yu J, Hu S. Mesenchymal stem cells microvesicles stabilize endothelial barrier function partly mediated by hepatocyte growth factor (HGF). Stem Cell Res Ther. 2017;8(1):211.

11. Pati S, Gerber MH, Menge TD, Wataha KA, Zhao Y, Baumgartner JA, Zhao J, Letourneau PA, Huby MP, Baer LA, et al. Bone marrow derived mesenchymal stem cells inhibit inflammation and preserve vascular endothelial integrity in the lungs after hemorrhagic shock. PLoS One. 2011;6(9):e25171.

12. Yu H, Xu Z, Qu G, Wang H, Lin L, Li X, Xie X, Lei Y, He X, Chen Y, et al. Hypoxic Preconditioning Enhances the Efficacy of Mesenchymal Stem Cells-Derived Conditioned Medium in Switching Microglia toward Anti-inflammatory Polarization in Ischemia/Reperfusion. Cell Mol Neurobiol. 2021;41(3):505-24.

13. García JR, Quirós M, Han WM, O'Leary MN, Cox GN, Nusrat A, García AJ. IFN-y-tethered hydrogels enhance mesenchymal stem cell-based immunomodulation and promote tissue repair. Biomaterials. 2019;220:119403.

14. Liu W, Yu M, Xie D, Wang L, Ye C, Zhu Q, Liu F, Yang L. Melatonin-stimulated MSC-derived exosomes improve diabetic wound healing through regulating macrophage M1 and M2 polarization by targeting the PTEN/AKT pathway. Stem Cell Res Ther. 2020;11(1):259.

15. Kim M, Yun HW, Park DY, Choi BH, Min BH. Three-Dimensional Spheroid Culture Increases Exosome Secretion from Mesenchymal Stem Cells. Tissue Eng Regen Med. 2018;15(4):427-36.

16. Pradhan G, Samson SL, Sun Y. Ghrelin: much more than a hunger hormone. Curr Opin Clin Nutr Metab Care. 2013;16(6):619-24.

17. Zheng H, Liang W, He W, Huang C, Chen Q, Yi H, Long L, Deng Y, Zeng M. Ghrelin attenuates sepsisinduced acute lung injury by inhibiting the NF-KB, iNOS, and Akt signaling in alveolar macrophages. Am J Physiol Lung Cell Mol Physiol. 2019;317(3):L381-I391. 
18. Han D, Huang W, Ma S, Chen J, Gao L, Liu T, Zhang R, Li X, Li C, Fan M, et al. Ghrelin improves functional survival of engrafted adipose-derived mesenchymal stem cells in ischemic heart through PI3K/Akt signaling pathway. Biomed Res Int. 2015;2015:858349.

19. Parhar KKS, Zjadewicz K, Soo A, Sutton A, Zjadewicz M, Doig L, Lam C, Ferland A, Niven DJ, Fiest KM, et al. Epidemiology, Mechanical Power, and 3-Year Outcomes in Acute Respiratory Distress Syndrome Patients Using Standardized Screening. An Observational Cohort Study. Ann Am Thorac Soc. 2019;16(10):1263-72.

20. Chen $\mathrm{H}$, Bai C, Wang X. The value of the lipopolysaccharide-induced acute lung injury model in respiratory medicine. Expert Rev Respir Med. 2010;4(6):773-83.

21. Hung SC, Pochampally RR, Chen SC, Hsu SC, Prockop DJ. Angiogenic effects of human multipotent stromal cell conditioned medium activate the PI3K-Akt pathway in hypoxic endothelial cells to inhibit apoptosis, increase survival, and stimulate angiogenesis. Stem Cells. 2007;25(9):2363-70.

22. Chang MC, Tsao $\mathrm{CH}$, Huang WH, Chih-Hsueh Chen P, Hung SC. Conditioned medium derived from mesenchymal stem cells overexpressing HPV16 E6E7 dramatically improves ischemic limb. J Mol Cell Cardiol. 2014;72:339-49.

23. Xu T, Lv Z, Chen Q, Guo M, Wang X, Huang F. Vascular endothelial growth factor over-expressed mesenchymal stem cells-conditioned media ameliorate palmitate-induced diabetic endothelial dysfunction through PI-3K/AKT/m-TOR/eNOS and p38/MAPK signaling pathway. Biomed Pharmacother. 2018;106:491-8.

24. Chen L, Xu Y, Zhao J, Zhang Z, Yang R, Xie J, Liu X, Qi S. Conditioned medium from hypoxic bone marrow-derived mesenchymal stem cells enhances wound healing in mice. PLoS One. 2014;9(4):e96161.

25. Ye N, Jiang D. Ghrelin accelerates the growth and osteogenic differentiation of rabbit mesenchymal stem cells through the ERK1/2 pathway. BMC Biotechnol. 2015;15:51.

26. Ye N, Wang L, Dou Z, Huang J. Ghrelin accelerates the cartilagic differentiation of rabbit mesenchymal stem cells through the ERK1/2 pathway. Cytotechnology. 2018;70(1):415-21.

27. Revathidevi S, Munirajan AK. Akt in cancer: Mediator and more. Semin Cancer Biol. 2019;59:80-91.

28. Huang B, Liu J, Meng T, Li Y, He D, Ran X, Chen G, Guo W, Kan X, Fu S, et al. Polydatin Prevents Lipopolysaccharide (LPS)-Induced Parkinson's Disease via Regulation of the AKT/GSK3 $\beta-N r f 2 / N F-$ KB Signaling Axis. Front Immunol. 2018;9:2527.

29. Si H, Zhang Y, Song Y, Li L. Overexpression of adrenomedullin protects mesenchymal stem cells against hypoxia and serum deprivation-induced apoptosis via the Akt/GSK3 $\beta$ and Bcl-2 signaling pathways. Int J Mol Med. 2018;41(6):3342-52.

30. Liu Y, Li JY, Chen ST, Huang HR, Cai H. The rLrp of Mycobacterium tuberculosis inhibits proinflammatory cytokine production and downregulates APC function in mouse macrophages via a TLR2-mediated PI3K/Akt pathway activation-dependent mechanism. Cell Mol Immunol. 2016;13(6):729-46. 
31. Qi D, Wang D, Zhang C, Tang X, He J, Zhao Y, Deng W, Deng X. Vaspin protects against LPS-induced ARDS by inhibiting inflammation, apoptosis and reactive oxygen species generation in pulmonary endothelial cells via the Akt/GSK-3ß pathway. Int J Mol Med. 2017;40(6):1803-17.

32. Qi Y, Zhang W, Li G, Niu L, Zhang Y, Tang R, Feng G. An oriented-collagen scaffold including Wnt5a promotes osteochondral regeneration and cartilage interface integration in a rabbit model. Faseb $\mathrm{j}$. 2020;34(8):11115-32.

33. Ni H, Ni T, Feng J, Bian T, Liu Y, Zhang J. Spondin-2 is a novel diagnostic biomarker for laryngeal squamous cell carcinoma. Pathol Res Pract. 2019;215(2):286-91.

34. Arcuri C, Bianchi R, Brozzi F, Donato R. S100B increases proliferation in PC12 neuronal cells and reduces their responsiveness to nerve growth factor via Akt activation. J Biol Chem. 2005;280(6):4402-14.

35. Kang MH, Kim JS, Seo JE, Oh SC, Yoo YA. BMP2 accelerates the motility and invasiveness of gastric cancer cells via activation of the phosphatidylinositol 3-kinase (PI3K)/Akt pathway. Exp Cell Res. 2010;316(1):24-37.

36. Yan Z, Yin H, Lin G. CircDDX42 Accelerates the Development of Pancreatic Cancer via miR613/ID4/PI3K/AKT Axis. Onco Targets Ther. 2020;13:10945-57.

37. Calvo N, Carriere P, Martín MJ, Gigola G, Gentili C. PTHrP treatment of colon cancer cells promotes tumor associated-angiogenesis by the effect of VEGF. Mol Cell Endocrinol. 2019;483:50-63.

38. Zhang S, Liu X, Ge LL, Li K, Sun Y, Wang F, Han Y, Sun C, Wang J, Jiang W, et al. Mesenchymal stromal cell-derived exosomes improve pulmonary hypertension through inhibition of pulmonary vascular remodeling. Respir Res. 2020;21(1):71.

39. Bretón-Romero R, Feng B, Holbrook M, Farb MG, Fetterman JL, Linder EA, Berk BD, Masaki N, Weisbrod RM, Inagaki E, et al. Endothelial Dysfunction in Human Diabetes Is Mediated by Wnt5a-JNK Signaling. Arterioscler Thromb Vasc Biol. 2016;36(3):561-9.

40. Michetti F, Corvino V, Geloso MC, Lattanzi W, Bernardini C, Serpero L, Gazzolo D. The S100B protein in biological fluids: more than a lifelong biomarker of brain distress. J Neurochem. 2012;120(5):64459.

41. Seguella L, Capuano R, Pesce M, Annunziata G, Pesce M, de Conno B, Sarnelli G, Aurino L, Esposito G. S100B Protein Stimulates Proliferation and Angiogenic Mediators Release through
RAGE/pAkt/mTOR Pathway in Human Colon Adenocarcinoma Caco-2 Cells. Int J Mo/ Sci 2019,
20(13).

42. Zuo WH, Zeng P, Chen X, Lu YJ, Li A, Wu JB. Promotive effects of bone morphogenetic protein 2 on angiogenesis in hepatocarcinoma via multiple signal pathways. Sci Rep. 2016;6:37499.

43. Fontemaggi G, Dell'Orso S, Trisciuoglio D, Shay T, Melucci E, Fazi F, Terrenato I, Mottolese M, Muti P, Domany $\mathrm{E}$, et al. The execution of the transcriptional axis mutant p53, E2F1 and ID4 promotes tumor neo-angiogenesis. Nat Struct Mol Biol. 2009;16(10):1086-93.

\section{Figures}




\section{Figure 1}

Rat BMSCs morphology and phenotype identification by flow cytometry. (a) BMSCs were isolated by the whole bone marrow adherence method and BMSCs from the third passage were used for subsequent experiments. (b d) For flow cytometry assay, P3 BMSCs have a positive expression of CD29 and CD90 and negative expression of CD45. Red solid lines represent the negative control while blue solid lines represent the expression of surface markers.

\section{Figure 2}

Selection of the optimum concentration and duration for ghrelin preconditioning. (a) BMSCs were cultured in serum-free medium containing gradient concentrations of ghrelin $(0,1,10,100,1000 \mathrm{nM})$, and optical density (OD) values at $450 \mathrm{~nm}$ were measured at $24,48,72$ hours after the culture with a microplate reader. (b) Endothelial cells were cultured in conditioned medium derived from BMSCs pretreated by gradient concentrations of ghrelin $(0,1,10,100,1000 \mathrm{nM})$ for 24 hours, and OD values at $450 \mathrm{~nm}$ were measured. According to the results, $100 \mathrm{nM}$ of ghrelin was an appropriate concentration for the subsequent experiments. Data were described with mean \pm standard deviation, $n=3 .{ }^{*} p<0.05,{ }^{*} p<0.01$ vs the control group. $\# p<0.05, \# \# p<0.01$ vs the LPS group,

\section{Figure 3}

BMSCsGhrelin pretreated-CM inhibited apoptosis and promoted migration of endothelial cells exposed to LPS. (a) Scratch assay was performed to evaluate the migration ability of endothelial cells, and representative pictures of scratches at different time points were shown. (b) Quantitative analysis for the changes of scratch areas was performed with Image J. The result of scratch assay revealed that ghrelin pretreatment promoted the pro-migration effect of BMSCs.(c) Mitochondrial membrane potential (MMP) was detected by JC-1 probe staining and (d) quantified by Image $\mathrm{J}$ using the ratio of red to green fluorescence. (e) Apoptotic rates were examined by flow cytometry using Annexin V-FITC and PI staining. (f) Quantitative analysis for the percentage of apoptotic cells. (g) Western blot analysis for pro-apoptotic protein Bax and anti-apoptotic protein Bcl-2. (h i) Semi-quantitative assay of Bax and Bcl-2 protein. The above results demonstrated a better anti-apoptotic ability of BMSCs pretrated by ghrelin. Data were described with mean \pm standard deviation, $n=3 .{ }^{*} p<0.05$, ${ }^{* \star} p<0.01$ vs the control group. $\# p<0.05, \# \# p<0.01$ vs the LPS group,

\section{Figure 4}


BMSCsGhrelin pretreated-CM reduced inflammation of endothelial cells with LPS treatment. The expression of inflammatory cytokines TNF-a, IL-1 $\beta$, and IL- 6 was detected by qPCR and shown in the bar graphs. The expression of the above cytokines were downregulated by both BMSCs-CM and BMSCsGhrelin pretreated-CM. Data were described with mean \pm standard deviation, $n=3 .{ }^{*} p<0.05$, ${ }^{* *} p<0.01$ vs the control group. $\# p<0.05, \# \# p<0.01$ vs the LPS group,

\section{Figure 5}

BMSCsGhrelin pretreated-CM activated the AKT/GSK3 $\beta$ pathway. (a) The expression of AKT, p-AKT, GSK3 $\beta$, p-GSK3 $\beta$ was detected by western blot and GAPDH was considered as the internal reference. $(b \sim c)$ The quantification of p-AKT and p-GSK3 $\beta$. (d f) Endothelial cells were treated with PI3K/AKT inhibitor LY294002 for 30 min before LPS and conditioned medium treatments, and the above proteins were detected and quantified again. The result of western blot demonstrated that BMSCsGhrelin pretreated-CM contributed to the activation of AKT/GSK3 $\beta$ pathway, which was reversed by LY294002. Data were described with mean \pm standard deviation, $n=3 .{ }^{*} p<0.05$, ${ }^{\star \star} p<0.01$ vs the control group. $\# p<0.05, \# \# p<0.01$ vs the LPS group,

\section{Figure 6}

AKT/GSK3 $\beta$ pathway was involved in the protective effects of BMSCsGhrelin pretreated-CM on endothelial cells. With LY294002, (a, c) the anti-apoptotic ability of Ghrelin pretreated BMSCs-CM was attenuated. (b, d) Scratch assay showed that LY294002 inhibited the migration of endothelial cells. (e f) The qPCR analysis indicated that LY294002 elevated the expression of pro-inflammatory factors. Data were described with mean \pm standard deviation, $n=3 .{ }^{*} p<0.05,{ }^{\star \star} p<0.01$ vs the control group. $\# p<0.05$, $\# \# p<0.01$ vs the LPS group,

\section{Figure 7}

RNA sequencing analysis for BMSCs pretreated by ghrelin. (a) Volcano plot of DEGs showed a total of 88 upregulated genes and 405 downregulated genes. (b, c) GO and KEGG analyses were performed to explore the interaction of DEGs. (d) Venn diagrams of upregulated DEGs. Unique and common DEGs among upregulated genes, genes associated with the activation of AKT pathway, and genes related to mediate the endothelial function were shown in the diagram. 